\title{
Introducing a Rheology Model for Non-Newtonian Drilling Fluids
}

\author{
Masoud Rashidi $\mathbb{D},{ }^{1}$ Ahmad Sedaghat $\mathbb{D},{ }^{2}$ Biltayib Misbah $\mathbb{D},{ }^{1}$ Mohammad Sabati $\mathbb{D},{ }^{3}$ \\ Koshy Vaidyan $\mathbb{D}^{1},{ }^{1}$ Ali Mostafaeipour ${ }^{(D},{ }^{4}$ Seyyed Shahabaddin Hosseini Dehshiri $\left(\mathbb{D},{ }^{5}\right.$ \\ Khalid Almutairi $\left(\mathbb{D},{ }^{6} \text { and Alibek Issakhov }{ }^{7}\right)^{7,8}$
}

\author{
${ }^{1}$ Petroleum Engineering Department, Australian College of Kuwait, Kuwait \\ ${ }^{2}$ Mechanical Engineering Department, Australian College of Kuwait, Kuwait \\ ${ }^{3}$ Electrical Engineering Department, Australian College of Kuwait, Kuwait \\ ${ }^{4}$ Industrial Engineering Department, Yazd University, Yazd, Iran \\ ${ }^{5}$ Department of Mechanical Engineering, Sharif University of Technology, Tehran, Iran \\ ${ }^{6}$ Applied College, Mechanical Engineering Technology, University of Hafr Al Batin, Hafr Al Batin, Saudi Arabia \\ ${ }^{7}$ Faculty of Mechanics and Mathematics, Department of Mathematical and Computer Modelling, Al-Farabi Kazakh \\ National University, Almaty 050040, Kazakhstan \\ ${ }^{8}$ Department of Mathematics and Cybernetics, Kazakh-British Technical University, Almaty 50000, Kazakhstan
}

Correspondence should be addressed to Ali Mostafaeipour; mostafaei@yazd.ac.ir

Received 23 May 2021; Revised 19 July 2021; Accepted 11 August 2021; Published 26 August 2021

Academic Editor: M.I. Herreros

Copyright (c) 2021 Masoud Rashidi et al. This is an open access article distributed under the Creative Commons Attribution License, which permits unrestricted use, distribution, and reproduction in any medium, provided the original work is properly cited.

\begin{abstract}
An API standard drilling fluid was investigated from laminar to turbulent flow conditions using an in-house-built viscometer at speeds from 200 to 1600 RPM. A power-based method was applied to obtain the apparent viscosity and the shear stress of the water-based drilling mud (WBM) in the annulus of the viscometer. Then, a MATLAB optimization program was developed to obtain model parameters for five rheology models integrated in a generalized Herschel-Bulkley-Extended (HBE) model and two widely used 4-parameter models in drilling industry. It is found that the Bingham, Cross, and HBE rheology models have precisely matched the WBM measurements in the viscometer. A generalized Reynolds number was applied to determine the Darcy friction factor although the PL (power law model) and the HB (Herschel-Bulkley model) exhibited a nonrealistic negative shift from the laminar friction factor.
\end{abstract}

\section{Introduction}

Energy is one of the most important needs of human life and plays an important role in the development of countries. Energy consumption is the amount of energy consumed in a year. It includes all energy harnessed from every energy source applied towards operation across all industrial and technical sectors, in every country [1]. World energy consumption has consequences for the socio-economic-political sphere $[2,3]$. Today, with the dramatic growth of population and the development of industries, the demand for energy in the world has increased $[4,5]$. Many countries are facing energy supply problems to meet the demand of the industrial, agricultural, and residential sectors [6]. The growing global demand for energy in many different countries [7] and the declining production of oil from existing oil sources have renewed engineers' interest in discovering new opportunities in deep and unconventional deep-water hydrocarbon reservoirs [8]. Extraction of crude oil from reservoirs is becoming more challenging due to various factors including increasing level of asphaltene, needs to drill deeper wells in search of new reservoirs, offshore explorations, and horizontal wellbore drilling. In tribology studies, the main attention is given to friction between two or more surfaces which is affected by asperity, abrasiveness, and shape of surfaces which are mainly dependent on materials of surfaces [9], whilst in rheology studies, 
behavior of fluids is sought. In oil and gas wells, drilling fluid exhibits shear thinning behavior which is known as thixotropy [10].

The drilling process is successful when the drilling fluid used in this process is effective [11]. The drilling method for oil and gas wells is drilling of a telescopic hole from the surface to the reservoir which can be kilometers away from the surface. Drilling is accomplished with the use of a drilling bit connected to a long string of drill pipe. By applying weight and rotation on the bit, the bit crushes the rock into small fragments, the cuttings $[11,12]$. Drilling fluid is circulated from the surface, through the drill pipe to the bit face; it lifts the generated cuttings and brings them to the surface, where separation equipment removes the cuttings from the drilling fluid, which is circulated, with the help of powerful pumps, back to the wellbore $[11,13]$. Drilling fluids perform additional functions [11, 13-15]; mainly, they control subsurface pressures, stabilize the exposed rock, prevent contamination of subsurface formation hydrocarbon fluids, provide buoyancy, and cool and lubricate the bit. Such fluids must be engineered so that they can perform efficiently in harsh environments, and it must be ensured that they do not damage the formations which are being drilled.

In oil and gas wellbore drilling industry, drilling fluids are highly complex and are prepared using certain additives to provide desirable tribology and rheology properties for specific drilling methods and reservoir conditions. The drilling fluids are generally classified as water-based mud (WBM), inhibitive WBM, oil-based mud (OBM), synthetic-based mud (SBM), foam fluids, gas or air, emulsions, and so on $[16,17]$. WBM fluids are preferred drilling fluids because of their environmentally friendly characteristics and lower operation costs, but OBMs are preferred fluids in deeper wellbore drilling and high-pressure-high-temperature (HPHT) drillings. This is partially attributed to the ionic nature of water molecules in WBM with a positive charge at the hydrogen side that attracts a negative charge at the oxygen side of water molecules by van der Waals forces. Hence, WBM is susceptible to higher internal friction as compared to OBM. Therefore, OBM drilling fluids are preferred over WBM fluids due to better lubrication properties.

The drilling industry is continuously seeking drilling fluids with enhanced mechanical, physical, chemical, and thermal properties that are also environmentally friendly in all exploration and extraction of oil and gas reservoirs [18]. To increase drilling speed and to overcome difficulties particularly in HPHT deep well drilling including increased drag force and torque, it is required to use "smart drilling liquids" that are usually referred to drilling fluids with additives such as organomolybdenum substances, organic friction reducers, and nanoparticles [19].

Fakoya and Ahmed [20] have studied rheological properties of WBM and OBM and developed generalized empirical models for apparent viscosity of OBM and WBM. Kumar et al. [21] suggested a generalized shear rate model for drilling fluids using FANN viscometer measurements. They claimed that the generalized shear rate model can be used for any rotational coaxial-cylinder viscometer with any fluids particularly Herschel-Buckley-type drilling fluids.
In well drilling operations, it is important to correctly determine the hydrodynamic behavior of drilling fluids in the well annulus space within "the operating window" to sustain correct pressure to avoid instability and fractural collapse of the well. The hydrodynamic nature of mud between the drill string and well bore is very complex due to the nonNewtonian nature of drilling fluid, laminar or turbulent flow condition, and eccentric rotation of the well string [22]. Classical lab equipment referred as the Taylor-Couette system (TCV) can be a good model for studying the wellbore drilling process. TCV consists of two coaxial rotational cylinders to study viscous flows in the gap of the system. For low rotational speed and low Reynolds number flows, the flow inside the gap is steady and azimuthal and is referred to as circular Couette flow. Couette [23] developed his system to be used as a viscometer. Later in 1923, Taylor [24] reported flow instability in TCV which opened the new science of hydrodynamic instability and proved the concept of no-slip condition on a wall. In general, TCV uses the inner cylinder as the rotating one and the outer one as the stationary one. Taylor developed linear stability theory by studying TCV with the inner cylinder being rotating and the outer cylinder being stationary. Taylor showed that the gap fluid flows become unstable if the rotational speed of the inner cylinder exceeds a particular limit.

TCV is one of the widely used geometries in turbulence modelling. The main advantages of TCV are that all power input of the system through an external electric motor is known and the energy loss on wall surfaces can be related to friction torque and rotational speed of TCV [25]. Many different flow regimes were observed in TCV including the Taylor vortex, modulated wavy vortex, wavy vortex, turbulent vortex, and random-pattern turbulent flows [26]. Many top scientists have studied Taylor-Couette flow regimes from laminar flow and its transition to instabilities and then to turbulence. Chandrasekhar [27] and Drazin and Reid [28] presented comprehensive theoretical solutions to many Taylor-Couette flow-type problems.

In the present work, a smaller Taylor-Couette viscometer (TCV) similar to [29] is designed and manufactured completely in Kuwait, with $100 \mathrm{ml}$ volume for fluids with circular speeds of $0-1600 \mathrm{rpm}$, to investigate experimentally skin friction of laminar and turbulent flows. Experimental measurements are conducted at different rotational speeds of the Taylor-Couette system. The WBM was tested at different rotational speeds of TCV. The experimental results in laminar and turbulent flow regimes of TCV are reported here.

\section{Research Methodology}

2.1. High-Speed Viscometer. The Taylor-Couette viscometer is a confined cavity between two cylinders with the same axis which are also closed from the top and the bottom by two disks as shown in Figure 1. Rotation of one or both cylinders creates a rotational confined viscous flow inside the gap of cylinders which are also influenced by the top and the bottom solid disk boundary conditions to make it a completely $3 \mathrm{D}$ complex flow system. Fluid flows in the TCV have been 


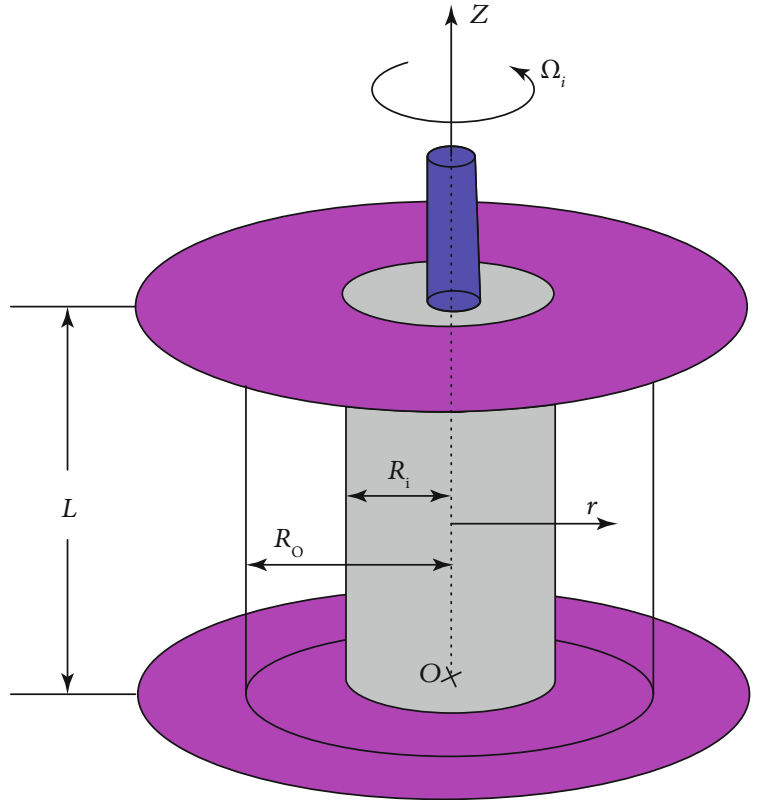

Figure 1: Sketch of the Taylor-Couette viscometer.

deeply investigated for a variety of flow regimes and flow patterns by many researchers.

Many complex flow phenomena have been observed in the Taylor-Couette system which is widely explored for studying linear and nonlinear hydrodynamic stability [30] and also for developing turbulence models [25]. Andereck et al. [26] reported a number of complex flow regimes for flows between two counterrotating cylinders in TCV such as basic Couette flow and appearance of Ekman cells, development of primary instabilities, wavy-vortex flow, low Reynolds modulated wavy-vortex flow, interpenetrating laminar spirals, wavy interpenetrating spirals, intermittency, transition region, spiral turbulence, turbulent flow, and hysteresis. For flow between corotating cylinders in TCV, they reported basic flow, primary instability, twisted vortices, wavy vortex boundary flows, and high azimuthal-wave number flows.

The most complete flow model for viscous flows is Navier-Stokes equations discussed next for TCV.

2.2. Taylor-Couette Flow Equations. The Navier-Stokes equations in three dimensions $(r, \theta, z)$ for incompressible viscous flows with constant dynamic viscosity $(\mu)$ and constant density $(\rho)$ may be expressed as follows [31]:

$$
\begin{gathered}
\frac{1}{r} \frac{\partial}{\partial r}\left(r u_{r}\right)+\frac{1}{r} \frac{\partial}{\partial \theta}\left(r u_{\theta}\right)+\frac{\partial u_{z}}{\partial z}=0 \\
\rho\left[\frac{D u_{r}}{D t}-\frac{u_{\theta}^{2}}{r}\right]=-\frac{\partial p}{\partial r}+F_{r}+\mu\left[\nabla^{2} u_{r}-\frac{u_{r}}{r^{2}}-\frac{2}{r^{2}} \frac{\partial u_{\theta}}{\partial \theta}\right], \\
\rho\left[\frac{D u_{\theta}}{D t}+\frac{u_{\theta} u_{r}}{r}\right]=-\frac{1}{r} \frac{\partial p}{\partial \theta}+F_{\theta}+\mu\left[\nabla^{2} u_{\theta}-\frac{u_{\theta}}{r^{2}}-\frac{2}{r^{2}} \frac{\partial u_{r}}{\partial \theta}\right],
\end{gathered}
$$

$$
\rho \frac{D u_{z}}{D t}=-\frac{\partial p}{\partial z}+F_{z}+\mu \nabla^{2} u_{z}
$$

In equations (1)-(4), $\left(u_{r}, u_{\theta}, u_{z}\right)$ and $\left(F_{r}, F_{\theta}, F_{z}\right)$ are velocity and body force components in $(r, \theta, z)$ directions, respectively. $p$ is the static or thermodynamic pressure, and the operators $D / D t$ and $\nabla^{2}$ are material derivative and Laplace operators defined by [31]

$$
\begin{aligned}
& \frac{D}{D t}=\frac{\partial}{\partial t}+u_{r} \frac{\partial}{\partial r}+\frac{u_{\theta}}{r} \frac{\partial}{\partial \theta}+u_{z} \frac{\partial}{\partial z}, \\
& \nabla^{2}=\frac{\partial^{2}}{\partial r^{2}}+\frac{1}{r} \frac{\partial}{\partial r}+\frac{1}{r^{2}} \frac{\partial^{2}}{\partial \theta^{2}}+\frac{\partial^{2}}{\partial z^{2}} .
\end{aligned}
$$

2.3. Stress Tensor in Newtonian Flows. For Newtonian incompressible flows, a stress tensor for an infinitesimal volume of fluid is obtained as follows [31]:

$$
\sigma_{r r}=-p+2 \mu \frac{\partial u_{r}}{\partial r}
$$

$$
\begin{gathered}
\sigma_{\theta \theta}=-p+2 \mu\left(\frac{1}{r} \frac{\partial u_{\theta}}{\partial \theta}+\frac{u_{r}}{r}\right), \\
\sigma_{z z}=-p+2 \mu \frac{\partial u_{z}}{\partial z}, \\
\sigma_{r \theta}=\mu\left(\frac{1}{r} \frac{\partial u_{r}}{\partial \theta}+\frac{\partial u_{\theta}}{\partial r}-\frac{u_{\theta}}{r}\right), \\
\sigma_{r z}=\mu\left(\frac{\partial u_{r}}{\partial z}+\frac{\partial u_{z}}{\partial r}\right), \\
\sigma_{\theta z}=\mu\left(\frac{1}{r} \frac{\partial u_{z}}{\partial \theta}+\frac{\partial u_{\theta}}{\partial z}\right) .
\end{gathered}
$$

2.3.1. Taylor-Couette System with the Stationary Outer Cylinder. Dou et al. [32] obtained solutions to the steadystate Navier-Stokes equation in TCV for incompressible Newtonian flows. For steady flows, the velocity component in the radial direction is assumed to be zero, i.e., $u_{r}=0$, and there are no changes in the tangential direction; hence, $\partial / \partial \theta=0$. Therefore, momentum equations in radial and circumferential directions (equations (2) and (3)) are reduced (by neglecting body forces) as follows [32]:

$$
\begin{gathered}
\rho \frac{u_{\theta}^{2}}{r}=\frac{\partial p}{\partial r}, \\
\frac{\partial}{\partial r}\left(\frac{\partial u_{\theta}}{\partial r}+\frac{u_{\theta}}{r}\right)=0 .
\end{gathered}
$$

Integrating equation (13) and considering boundary conditions at walls of inner and outer cylinders as follows:

$$
\begin{aligned}
& r=R_{\mathrm{i}}, u_{\theta}=R_{\mathrm{i}} \Omega_{\mathrm{i}}, \\
& r=R_{\mathrm{o}}, u_{\theta}=R_{\mathrm{o}} \Omega_{\mathrm{o}},
\end{aligned}
$$


and using $\chi=R_{\mathrm{i}} / R_{\mathrm{o}}$ and $\psi=\Omega_{\mathrm{o}} / \Omega_{\mathrm{i}}$, in which $\Omega_{\mathrm{i}}$ and $\Omega_{\mathrm{o}}$ are the rotational speeds of the inner and outer cylinders, respectively, the solution for velocity in the circumstantial direction is obtained as [32]

$$
u_{\theta}=A_{\theta} r+\frac{B_{\theta}}{r} .
$$

Parameters $A_{\theta}$ and $B_{\theta}$ are provided as [32]

$$
\begin{gathered}
A_{\theta}=\Omega_{i} \frac{\chi^{2}-\psi}{\chi^{2}-1}, \\
B_{\theta}=\Omega_{\mathrm{i}} R_{\mathrm{i}}^{2} \frac{1-\psi}{1-\chi^{2}} .
\end{gathered}
$$

The shear stress on the infinitesimal element shown in Figure 2 may be extended and rewritten for nonNewtonian and turbulent flows using equation (9) as follows [32]:

$$
\tau=\sigma_{r \theta}=\eta\left(\frac{\partial u_{\theta}}{\partial r}-\frac{u_{\theta}}{r}\right)
$$

where $\eta$ is the apparent viscosity provided in Table 1 and at the limit of Newtonian laminar flow reduces to dynamic viscosity $(\mu)$. Substituting the tangential velocity from equation (15) into equation (18), one may obtain

$$
\tau=\eta\left[\left(A_{\theta}-\frac{B_{\theta}}{r^{2}}\right)-\frac{1}{r}\left(A_{\theta} r+\frac{B_{\theta}}{r}\right)\right]=-2 \eta \frac{B_{\theta}}{r^{2}} .
$$

For TCV with the stationary outer cylinder $(\psi=0)$, the wall shear rate $\dot{\gamma}_{\text {wi }}$ at $r=R_{\mathrm{i}}$ is obtained using equations (17) and (19) as follows:

$$
\dot{\gamma}_{\mathrm{wi}}=\frac{\tau_{\mathrm{wi}}}{\eta_{\mathrm{wi}}}=\frac{2 B_{\theta}}{R_{\mathrm{i}}^{2}}=\frac{2 \Omega_{\mathrm{i}}}{1-\chi^{2}}=\frac{2 \Omega_{\mathrm{i}} R_{\mathrm{o}}^{2}}{R_{\mathrm{o}}^{2}-R_{\mathrm{i}}^{2}} .
$$

The $\eta_{\mathrm{wi}}$ in equation (20) is the apparent viscosity.

In the ACK TCV in steady rotation, the input electric power to the DC motor is divided into two components: (1) mechanical power formed by friction between rotating cylinders and the bearing system $\left(P_{\text {mech }}=T_{\text {mech }} \Omega_{\mathrm{i}}\right)$ and (2) fluid power developed by viscous effects of fluid on wet surfaces of the inner cylinder $\left(P_{\text {fluids }}=T_{\text {fluids }} \Omega_{\mathrm{i}}\right)$. The mechanical power $\left(P_{\text {mech }}\right)$ can be easily measured by running TCV without any fluid at different speeds. The fluid power is then obtained by subtracting the reading total power from the frictional power, i.e., $P_{\text {fluids }}=P_{\text {tot }}-P_{\text {mech }}$, at the same speed $\left(\Omega_{\mathrm{i}}\right)$. The total torque applied on the inner cylinder of TCV similarly consists of the mechanical torque from frictions on bearings and the torque from fluid viscosity and turbulence effects. Hence, the torque from fluid is obtained as follows:

$$
T_{\text {Fluids }}=\frac{P_{\text {Fluids }}}{\Omega_{\mathrm{i}}} .
$$

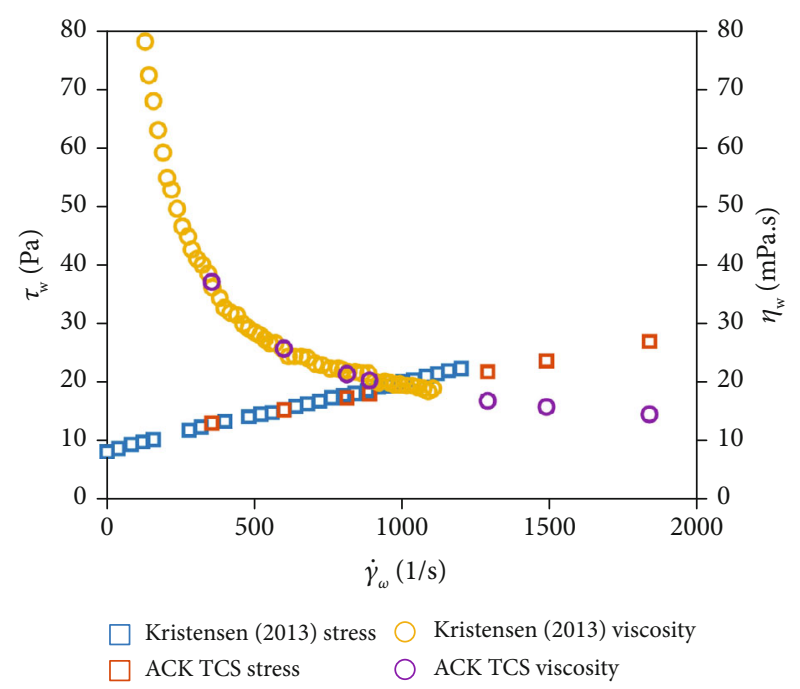

Figure 2: Calibration of the ACK TCS versus an Anton Paar MCR302 rheometer data [33].

The shear stress developed from fluid on the wall $\left(\tau_{\mathrm{wi}}\right)$ can be obtained by the fluid torque obtained from equation (21) divided by $R_{\mathrm{i}}$ and then divided by the inner cylinder circumstantial area $\left(A=2 \pi R_{\mathrm{i}} L\right)$, where $L$ is the length of the cylinder, as follows:

$$
\tau_{\mathrm{wi}}=\frac{T_{\text {Fluids }}}{2 \pi R_{\mathrm{i}}^{2} L} .
$$

Equation (22) is valid for Newtonian, laminar, and steady flows. For non-Newtonian fluids and turbulent flow condition, a calibration factor $\left(\tau_{\text {wi-non-Newtonian }}=\beta \tau_{\text {wi }}\right)$ was used in which the calibration factor $\beta$ is obtained by fitting the TCV experimental data versus a high-precision rheometer. Hence, the apparent viscosity of the fluid on the wall of the inner cylinder of TCV can then be determined using equations (20) and (22) by

$$
\eta_{\mathrm{wi}}=\frac{\tau_{\mathrm{wi}}}{\dot{\gamma}_{\mathrm{wi}}}=\frac{T_{\text {Fluids }}}{2 \pi R_{\mathrm{i}}^{2} L} \frac{\left(1-\chi^{2}\right)}{2 \Omega_{\mathrm{i}}}=\frac{T_{\text {Fluids }}\left(R_{\mathrm{o}}^{2}-R_{\mathrm{i}}^{2}\right)}{4 \pi R_{\mathrm{i}}^{2} L \Omega_{\mathrm{i}} R_{\mathrm{o}}^{2}} .
$$

In equation (23), $\eta_{\mathrm{wi}}$ is valid for both Newtonian and non-Newtonian fluids. For turbulent flows, the apparent viscosity consists of laminar and turbulent components $\left(\eta_{\text {wi }}\right.$ $\left.=\eta_{\text {wi-laminar }}+\eta_{\text {wi-turbulent }}\right)$.

Based on [10], we can use the same formulations developed above for Newtonian fluids, i.e., shear stress and shear rate in TCV, for non-Newtonian fluids. However, for consistency curves, we should use formulations presented in Table 1. More rheological models may be found in other sources $[10,17]$.

2.4. Flow Characteristics in TCV. The Reynolds number is a widely used factor for distinguishing different flow regimes in internal and external flows from laminar to turbulent conditions. In TCV, the Reynolds number is usually defined based on inner or outer cylinder linear velocities; however, 
TABLE 1: Generalized Reynolds number definitions for non-Newtonian fluids [34].

\begin{tabular}{|c|c|}
\hline Non-Newtonian fluids & Generalized Reynolds number \\
\hline \multirow{3}{*}{ Herschel-Bulkley-Extended (HBE) } & $\eta=\left(\tau_{0} / \dot{\gamma}\right)+K \dot{\gamma}^{n-1}+\eta_{\infty}$ \\
\hline & $\operatorname{Re}_{\operatorname{gen~HBE}}=\rho D^{n} \bar{u}^{2-n} / \tau_{0} / 8(D / \bar{u})^{n}+K[(3 m+1) / 4 m]^{n} 8^{n-1}+\eta_{\infty}(3 m+1) / 4 m(D / \bar{u})^{n-1}$ \\
\hline & $m=n K(8 \bar{u} / D)^{n}+\eta_{\infty}(8 \bar{u} / D) / \tau_{0}+K(8 \bar{u} / D)^{n}+\eta_{\infty}(8 \bar{u} / D)$ \\
\hline \multirow{3}{*}{ Herschel-Bulkley (HB) } & $\eta_{\infty}=0 ; \eta=\left(\tau_{0} / \dot{\gamma}\right)+K \dot{\gamma}^{n-1}$ \\
\hline & $\operatorname{Re}_{\operatorname{gen~HB}}=\rho D^{n} \bar{u}^{2-n} / \tau_{0} / 8(D / \bar{u})^{n}+K[(3 m+1) / 4 m]^{n} 8^{n-1}$ \\
\hline & $m=n K(8 \bar{u} / D)^{n} / \tau_{0}+K(8 \bar{u} / D)^{n}$ \\
\hline \multirow{2}{*}{ Power law (PL) } & $\tau_{0}=0 ; \eta_{\infty}=0 ; \eta=K \dot{\gamma}^{n-1} ; m=n$ \\
\hline & $\operatorname{Re}_{\text {gen PL }}=\rho D^{n} \bar{u}^{2-n} / K[(3 m+1) / 4 m]^{n} 8^{n-1}$ \\
\hline \multirow{3}{*}{ Bingham } & $K=0 ; n=1 ; \eta=\left(\tau_{0} / \dot{\gamma}\right)+\eta_{\infty}$ \\
\hline & $\operatorname{Re}_{\text {gen Bingham }}=\rho D \bar{u} / \tau_{0} / 8(D / \bar{u})+\eta_{\infty}(3 m+1) / 4 m$ \\
\hline & $m=\eta_{\infty}(8 \bar{u} / D) / \tau_{0}+\eta_{\infty}(8 \bar{u} / D)$ \\
\hline \multirow{2}{*}{ Newtonian } & $\tau_{0}=0 ; K=0 ; \eta=\eta_{\infty} ; n=m=1$ \\
\hline & $\operatorname{Re}_{\text {Newtonian }}=\rho D \bar{u} / \eta_{\infty}$ \\
\hline
\end{tabular}

the better definition is used based on the average velocity in the gap of TCV. Hence, for Newtonian incompressible and steady flows in TCV, the Reynolds number (Re) is defined here as follows:

$$
\operatorname{Re}=\frac{\bar{u}_{\theta} \delta}{v} .
$$

In equation $(24), \bar{u}_{\theta}(\mathrm{m} / \mathrm{s})$ is the average circular speed in the cavity of TCV and $\delta\left(=R_{\mathrm{o}}-R_{\mathrm{i}}\right)(\mathrm{m})$ is the gap distance between two cylinders (the length scale), and $v=\eta / \rho\left(\mathrm{m}^{2} / \mathrm{s}\right)$. The generalized Reynolds number is presented in Table 1 for different Newtonian/non-Newtonian fluids. Based on Table 1 , the average velocity in the gap should be applied using the velocity profile in the gap (equation (15)) as follows:

$$
\bar{u}_{\theta}=\frac{1}{\delta} \int_{R_{\mathrm{i}}}^{R_{\mathrm{o}}} u_{\theta} d r=\frac{B_{\theta}}{\delta} \ln \left(\frac{R_{\mathrm{o}}}{R_{\mathrm{i}}}\right)+\frac{A_{\theta}}{2 \delta}\left(R_{\mathrm{o}}^{2}-R_{\mathrm{i}}^{2}\right) .
$$

For fluids in Table 1 , the average circular speed $\bar{u}_{\theta}$ is given by equation (25) and the gap length $\delta$ is used instead of $D$ in the Reynolds definitions.

TCV may be used to investigate the friction factor of laminar and turbulent flows; therefore, it is more appropriate to use a dimensionless parameter, i.e., the Darcy friction factor, instead of shear stress on the wall. In TCV, the Darcy friction factor is given by [34]

$$
f_{\text {Darcy }}=\frac{8 \tau_{\mathrm{wi}}}{\rho\left(\bar{u}_{\theta}\right)^{2}} .
$$

In equation (26), $\rho$ is the fluid density, $\tau_{\mathrm{wi}}$ is the wall shear stress obtained from measured values of fluid torque using equation (22), and $\bar{u}_{\theta}$ is the average velocity in the gap of TCV given in equation (25). The Darcy friction factor in equation (26) is valid for any type of fluids from Newtonian to non-Newtonian and any flow regime from laminar to turbulent. In order to assess validity of experimental values of the ACK TCV against the widely accepted theoretically and experimentally validated friction factor in laminar flows, the laminar friction factor is defined and used as follows [34]:

$$
f_{\text {laminar }}=\frac{64}{\operatorname{Re}_{\mathrm{Gen}}} .
$$

In equation (27), the generalized Reynolds number $\left(\operatorname{Re}_{\mathrm{Gen}}\right)$ is obtained from Table 1 for various fluid types.

\section{Drilling Process}

The drilling process in an oil and gas industry involves using a drilling rig which rotates a drill string with a drill bit attached. Drilling is achieved by using the drill bit that is attached to a long string of the drill pipe. Digging a wellbore may require drilling from the ground surface to reach deep into an oil or gas reservoir. The weight of a drilling rig on the bit in addition to the rotation of the bit cuts soil and rocks into smaller pieces and transports them out through a fluid circulating system. The drilling process is highly dependent on the performance of circulating well drilling fluids. Drilling fluids are usually WBM or OBM which are pumped from a mud tank into the drilling pipe inside the wellbore and back to the mud tank. The drilling fluids then transport mud and rocks through the cavity space between the drilling pipe and the wellbore back to the ground. A separator device at the ground is used to separate shale pieces from the drilling fluid. The circulating mud system uses a 
powerful pump to circulate the mud into and from the wellbore.

3.1. Functions of Drilling Mud. One of the important factors in wellbore drilling performance is drilling fluids. Drilling liquids are specifically designed to maximize wellbore drilling performances, to minimize risks to drilling rig operations and equipment, and to be safe to the environment. From the economic point of view, it is important to highlight that the wellbore drilling process counts between 20 and 40 percent of the total cost. Therefore, the drilling fluids are needed to be continuously enhanced to supply the following functions in wellbore drilling:

(1) Transport mud and rocks by the drilling bit back into the surface

(2) Drilling mud provides suitable subsurface pressure for sustainable shale during drilling operation

(3) Lubricate the drill string and remove heat generated because of the function of the bit and friction through mechanical movement of the drill string against the wellbore and hydraulic systems

(4) Support sustainable and stable formation of wellbore shale [35]

OBMs are commonly used in wellbore drilling at HPHT, but OBMs are expensive and not environmentally friendly. WBMs are less expensive and safe to the environment yet are limited to minimal temperatures and pressures and are more susceptible to breakdown, gel formation, and degradation of shale soft materials if exposed to high temperatures [35]. Therefore, it is continuously needed to modify wellbore drilling technology and enhance OBM and WBM properties to improve the life cycle of drilling equipment and to reduce costs. We only considered WBM in this research mainly because of environment-friendly aspects, lower costs, and more stability compared to the OBM.

3.2. Drilling Fluid Properties. In order to enhance drilling mud properties for efficient and safe operation of wellbore drilling, four primary properties including density, viscosity, gel strength, and filtration must be improved [35]. Gel strength is simply referred to as the shear stress of drilling mud at rest. The gel strength is important to withhold the shale formation when the drilling operations stop. Filtration is a measure of how much drilling fluids are recovered after separating from shale materials. Viscosity is basically referred to as fluid internal resistance and is obtained by the ratio of shear stress to shear strain. A diagram that shows the relationship between shear stress and shear strain is referred to as the consistency chart. Figure 3 shows the general consistency chart for different fluids. Rheological terminology used for drilling fluid can be seen in Figure 3, which distinguishes Newtonian and non-Newtonian fluids and provides graphical description of the yield point (YP), plastic viscosity (PV), and typical drilling mud behavior.

In general, lower viscosity or lower fluid friction of mud fluids provides more shale stability and less fluid loss. In

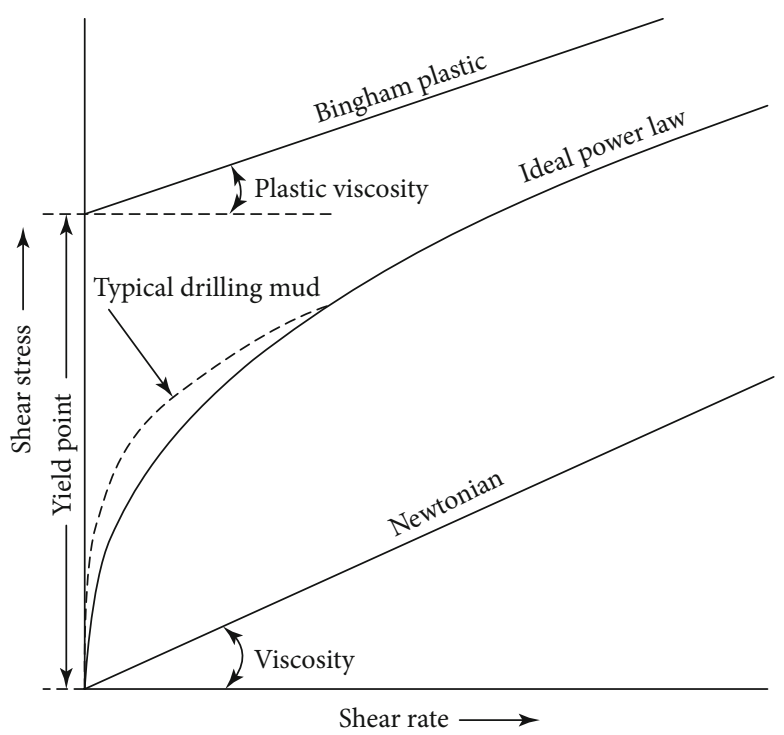

FIgURE 3: Consistency chart for Newtonian and non-Newtonian fluids (typical drilling muds) [35].

addition, lower viscosity (thinner fluids) provides better cleaning of bits and improves drilling performance; however, thicker mud fluids are sometimes required to extract larger gravel out of the wellbore during drilling operations.

\section{Rheology of Drilling Muds}

The classical rheological models for drilling fluids shown in Figure 3 are extended by Shah et al. [17] to include a wider classification such as non-Newtonian pseudoplastic and yield pseudoplastic fluids. Summary of the models is presented in Table 1.

Fakoya and Ahmed [20] developed more general empirical models than those shown in Table 1 for viscosity of OBM drilling fluids by considering temperature, pressure, volume concentration, and other factors. Kumar et al. [21] also provided theoretically a very complex model for estimation of shear rates.

4.1. Generalized Reynolds Number. In non-Newtonian fluid flows, the relation between shear stress and shear strain is nonlinear and the apparent viscosity $(\eta=\tau / \rho)$ is no longer constant. Depending on the apparent viscosity, nonNewtonian fluids are classified as follows [10]:

(1) Pseudoplastic or shear thinning

(2) Dilatant or shear thickening

(3) Viscoplastic or yield pseudoplastic or Bingham plastic

For analyzing non-Newtonian fluids, the Reynolds number, however, has to be redefined depending on different types of non-Newtonian fluids. Many different models were used for various flow types such as Herschel-Bulkley- (HB-), Ostwald-de Waele- (power law (PL)), and Bingham-type flows. Madlener et al. [34] introduced a new definition for 
the Reynolds number in non-Newtonian media. Table 1 illustrates various Reynolds number definitions for different non-Newtonian flows [34].

In Table $1, \rho, D, \bar{u}, \eta$, and $\eta_{\infty}$ are the density, the length scale (diameter for pipes), the average velocity, the variable apparent viscosity as a function of shear rate, and the infinite viscosity, respectively. $K$ and $n$ are consistency parameters, $n$ is also called the global exponential term, $m$ is a local term, $\tau_{0}$ is the yield stress, and $\dot{\gamma}$ is the shear rate (strain rate).

4.2. Four-Parameter Models. The more common fourparameter models for drilling fluids are Cross and Carreau models described by [17]

$$
\begin{array}{r}
\text { Cross Model }: \eta=\eta_{\infty}+\frac{\left(\eta_{o}-\eta_{\infty}\right)}{\left(1+(K \dot{\gamma})^{n}\right)}, \\
\text { Carreau Model }: \eta=\eta_{\infty}+\frac{\left(\eta_{o}-\eta_{\infty}\right)}{\left(1+(K \dot{\gamma})^{2}\right)^{-n / 2}} .
\end{array}
$$

These models are also considered here for modelling WBM measurements.

4.3. Optimization Technique. As illustrated in Table 1 and Section 4.2 , the apparent viscosity is expressed by a function of shear rate $\left(\dot{\gamma}_{\mathrm{wi}}\right)$ described by rheology models with maximum four unknown constants, e.g., HBE and Cross models, as follows:

$$
\eta_{\mathrm{wi}}=f\left(\dot{\gamma}_{\mathrm{wi}}, K, n, \tau_{0} \text { or } \eta_{\mathrm{o}}, \eta_{\infty}\right)
$$

The formulations given in the previous sections are used to obtain 4 unknown constants in equation (30) for WBM studied here. To determine 4 unknowns based on the data collected from the ACK TCV and formulations given in the previous sections, an optimization method is used to fit the best function to measured data. To fit model parameters given in equation (30) with measured data, the optimization toolbox "fminsearch" in MATLAB was used which uses a regression method to find optimization variables by minimizing an objective function. The objective function used here utilizes a root mean square method based on $R^{2}$. The routine code in MATLAB is presented in Algorithm 1.

4.4. Convergence Criteria. The convergence criteria use $R^{2}$ to measure the accuracy of the predicted and actual data. We used the following relation to be minimized as the objective function in MATLAB for predicted values "Pred" and measured data for the apparent viscosity (AV) as follows [36, 37]:

$$
\text { Objective function }=1-R^{2}=\frac{\sum(\text { Pred }-\mathrm{AV})^{2}}{\sum(\mathrm{AV}-\overline{\mathrm{AV}})^{2}} .
$$

Equation (31) presents $\overline{\mathrm{AV}}$ as the average viscosity, $\mathrm{AV}$ as the measured value, and "Pred" as the predicted model value. The $R^{2}$ close to one gives the highest accuracy; there-

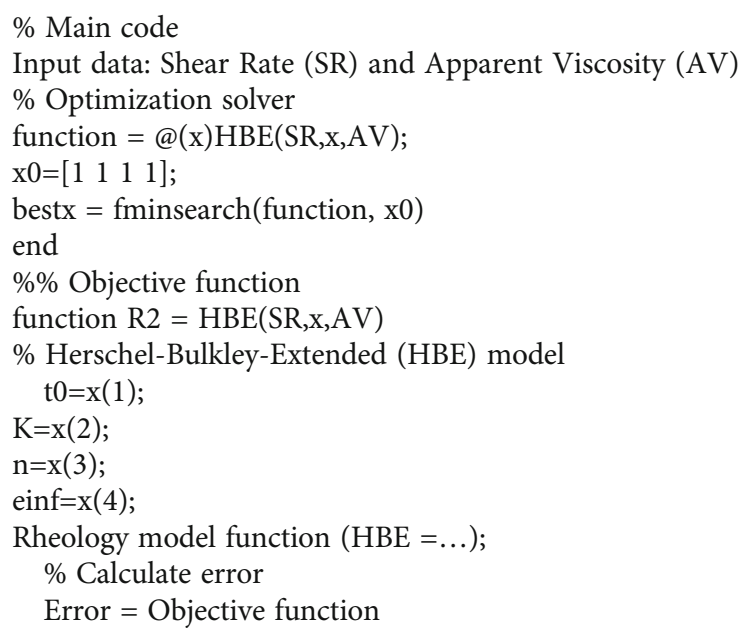

Algorithm 1: Optimization routine in MATLAB.

fore, Objective function $=1-R^{2}$ merges to zero using the optimization method shown in Algorithm 1.

\section{Experimental Setup}

5.1. TCV Apparatus. The Taylor-Couette viscometer (TCV) consists of two cylinders with the same axis where the outer cylinder is the stator and the inner one is the rotor connected to an electric motor rotating with constant speed. A similar design to the TCV by Dr. Daniel Borrero Echeverry [29] at Willamette University was developed but with smaller size, different drive, and in-house-developed control system. In the ACK TCV shown in Figure 4, the inner cylinder is seated on two sets of the bearing system at the top and bottom of TCV. The outer cylinder is made from acrylic and the inner cylinder from stainless steel. The gap between acrylic and stainless-steel cylinders is filled in with fluids at a hole in the top, and after the experiment, the fluid is discharged from an elastic hose and valve at the bottom. In the TCV design of Willamette University, a stepper motor is used to rotate the inner cylinder. Technical details of the TCV components may be obtained from [29].

Due to simplicity and safe operation, the TaylorCouette system is an ideal device for studying various flow patterns and flow instabilities. Moreover, TCV is an outstanding platform to observe various flow patterns. At low speeds, the fluid particles follow circular paths and have no dependency on axial nor azimuthal directions. As rotational speed increases, the flow transition is initiated. At transitional flow condition, instability patterns such as cellular flow type and toroidal vortices appeared. These vortices are called Taylor rolls. By increasing the speed of the inner cylinder, these Taylor rolls become more unstable and create very complex flow patterns, and then, the flow becomes turbulent. Table 2 provides the main features and geometry of the ACK TCV.

Figure 4 shows the manufactured TCV of ACK in Kuwait. We used a DC motor which enables us to run 


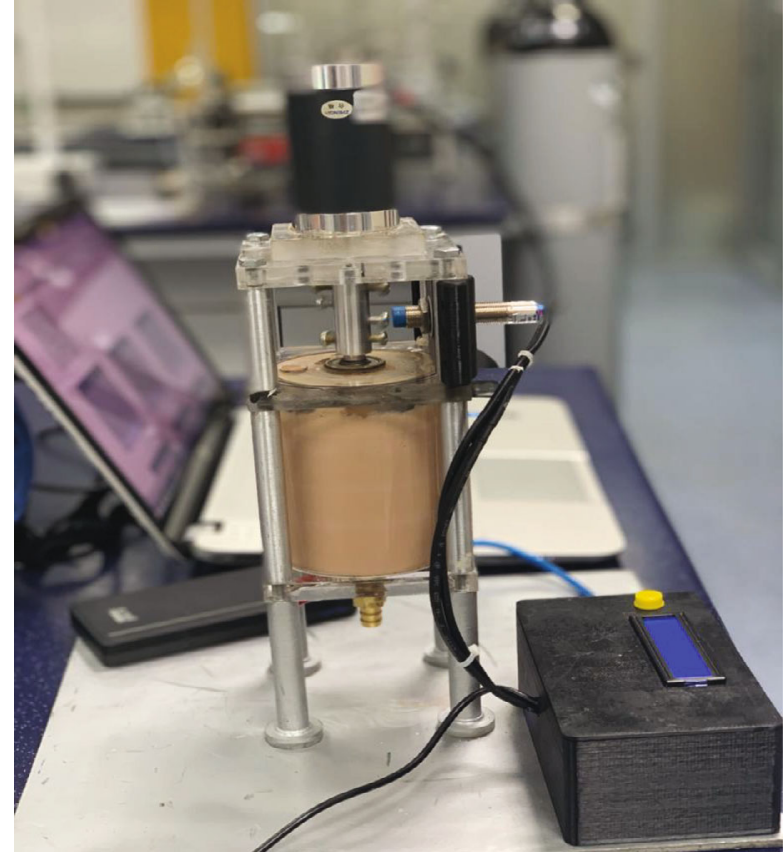

FIGURE 4: Taylor-Couette system of ACK for studying rheology of drilling nanofluids with major dimensions: inner radius $R_{\mathrm{i}}=3.4$ $\mathrm{cm}$, outer radius $R_{\mathrm{o}}=3.8 \mathrm{~cm}$, length $L=11.0 \mathrm{~cm}$, gap $\delta=R_{\mathrm{o}}-R_{\mathrm{i}}$ $=0.4 \mathrm{~cm}$, and annulus volume of $\forall=\pi\left(R_{\mathrm{o}}^{2}-R_{\mathrm{i}}^{2}\right) L=100 \mathrm{ml}$ [38, 39].

TCV at different speeds from 0 to $1600 \mathrm{rpm}$ and is also easy to measure electric power. The DC motor uses a speed controller which also displays motor speed. The TCV of ACK uses a controller box which uses two Arduino microprocessors, a tachometer, and a rheostat to measure voltage and current of the DC electric motor and angular velocity and to change the speed of the motor, respectively. The DC electric motor rotates at different speeds from 0 to $1600 \mathrm{rpm}$. We have used the TCV for testing WBM (at room temperature) at speeds from $200 \mathrm{rpm}$ to $1600 \mathrm{rpm}$ with intervals of $200 \mathrm{rpm}$. The DC electric motor is connected to a rheostat (variable resistance) to control the speed, and it is also connected with two Arduino microprocessors: one is used to measure voltage and the other one to measure the current supplied to the motor. A tachometer is mounted on the side of the device to measure angular velocity of the shaft of the DC motor. Two Arduino microprocessors are programmed to process all sensor measurements and display RPM, $\mathrm{rad} / \mathrm{s}$, power (W), and viscosity (cp) on an LCD $16 \mathrm{~cm} \times 2 \mathrm{~cm}$ screen. The controller box is produced using $3 \mathrm{D}$ prints.

At steady-state rotation and turbulent flows, measuring the power and torque exerted by the fluid on the inner cylinder is the best candidate for evaluating fluid resistance in non-Newtonian turbulent flows from a practical point of view.

5.2. WBM Preparation. WBMs are prepared using water as the base fluid and several soluble substances such as salts, surfactants, alkalies, polymers, and oils and several insoluble elements such as clay, cuttings, and barite in suspension.
Several WBM types have already been used in well drilling industry such as salt, lime, spud, deflocculated substance, gypsum, polymer, potassium, metal hydroxide, and cationic muds [40]. In literature, various improvements are applied to enhance viscosity, yield point, gel, and thermal stability by carefully selecting different components and composition [40].

Water-based fluids are prepared in the labs according to the "pilot testing" which is proportioned to small-scale samples based on using $1 \mathrm{~g} / 350 \mathrm{ml}$ of the sample (equivalent to $1 \mathrm{lb} / \mathrm{bbl}(42 \mathrm{gal})$ ) of the actual mud system. The WBM sample is prepared by adding distilled (deionized) water and the additives as follows [41]:

(1) Sodium carbonate (soda ash) as a hardener agent $(0.2-0.5 \mathrm{~g})$

(2) Sodium hydroxide (caustic soda) as a $\mathrm{pH}$ controller $(0.25-0.3 \mathrm{~g})$ to give $\mathrm{pH} 9-10$

(3) Bentonite (10-20 g) as a viscous agent

(4) Starch as a filtration loss controller

(5) Barite as a weighing agent

In this work, we prepared drilling WBM based on the international standard API RP 131 [42]. In the API RP 131 standard, distilled or deionized water is prepared based on ISO 3696:1987 or prepared by passing distilled water through a series of cation and anion exchange resins [42]. Calibration bentonite is obtained according to API 13A. The standard WBM is prepared according to the following steps:

(1) At a room temperature, $15 \mathrm{~g}$ of bentonite is mixed with $350 \mathrm{ml}$ of deionized water in a mud mixer $(4 \%$ vol bentonite API standard)

(2) At every 5 minutes, mud on the surfaces of the mixer should be dissolved

(3) The above two steps should be repeated for 20minute duration

The same procedure mentioned above was applied in all our WBM preparations.

\section{Results of Measurements}

6.1. Power Consumption. The ACK TCV uses power consumptions to determine rheology. To take into account frictional power loss, the TCV was first calibrated to measure power loss due to mechanical friction. Then, WBM was filled up into the TCV and power consumptions were measured. The TCV reading was conducted by allowing a steady state which is reached as each speed.

Unlike stepper motors, the speed controller of the DC motor used cannot provide a fixed preset value for speed. Thus, we used the best fit, to the raw data given in Table 3 , to obtain power values at precise rotational speed of TCV. Figure 5 compares mechanical power (air), total power 
TABLE 2: Main specification and sizes of the ACK TCV viscometer.

\begin{tabular}{lc}
\hline Parameters & Values \\
\hline Inner cylinder radius $\left(R_{\mathrm{i}}\right)$ & $34 \mathrm{~mm}$ \\
Outer cylinder radius $\left(R_{\mathrm{o}}\right)$ & $38 \mathrm{~mm}$ \\
Gap width $(\delta)$ & $4 \mathrm{~mm}$ \\
Wet length of the cylinder $(L)$ & $110 \mathrm{~mm}$ \\
Wet annulus volume $(\forall)$ & $100 \mathrm{ml}$ \\
Thickness of the outer cylinder & $3 \mathrm{~mm}$ \\
Electric DC motor speed range & 0 to $1600 \mathrm{RPM}$ \\
Material of the inner cylinder & Stainless steel \\
Material of the outer cylinder & Acrylic \\
\hline
\end{tabular}

(fluid+air), and fluid power for testing TCV without liquid and with WBM.

As observed in Figure 5, the mechanical power (W) of $\mathrm{TCV}$ is a linear function of angular speed $(\mathrm{rad} / \mathrm{s})$ for air as follows:

$$
P_{\text {mech }}=0.1128 \Omega_{\mathrm{i}} .
$$

Figure 5 also shows that the base fluid, i.e., WBM, is a second-ordered polynomial function of rotational speed $(\mathrm{rad} / \mathrm{s})$ :

$$
P_{\text {fluid }}=0.0013 \Omega_{\mathrm{i}}^{2}+0.1553 \Omega_{\mathrm{i}} \text {. }
$$

$R^{2}$ accuracy of the fitted functions is very closed to 1 , so these are highly accurate. Mechanical power (air) given in equation (30) should be deducted from the total power measurements of WBM to obtain net fluid power using equation (31).

6.2. Flow Properties. Flow properties in the ACK TCV including the apparent viscosity, shear stress, shear rate, average velocity, Reynolds number, and Darcy friction factor are obtained from the measurements of the power and the rotational speed and the outlined formulations given in Research Methodology. The results of measurements are listed in Table 3 for WBM in the ACK TCV.

6.3. Calibration of the ACK TCV. Results of WBM flow in the ACK TCV are presented in Table 3 for speeds 01600 RPM (200 RPM intervals). First, we calibrated the TCV using high-precision rheometer measurements reported in [33], and the comparison of the viscosity and stress is shown in Figure 2.

We investigate the accuracy of these measurements by fitting different rheology models presented in Table 1.

6.3.1. Newtonian Rheology Model. In the Newtonian rheology (Table 1), it is assumed that $\tau_{0}=0, K=0, \eta=\eta_{\infty}, n=m$ $=1$, and $\operatorname{Re}_{\text {Newtonian }}=\rho D \bar{u} / \eta_{\infty}$. Therefore, the model (equation (28)) needs only one parameter, i.e., $\eta_{\infty}$. Having applied the optimization program in MATLAB, we compare rheology model parameters with the experimental data (Table 4).
TABle 3: Power measurements of TCV for air and WBM.

\begin{tabular}{lccc}
\hline & Air & \multicolumn{2}{c}{ WBM } \\
Speed $(\mathrm{rad} / \mathrm{s})$ & Power $(\mathrm{W})$ & Speed $(\mathrm{rad} / \mathrm{s})$ & Power $(\mathrm{W})$ \\
\hline 24.63 & 3.22 & 21.68 & 7.89 \\
50.35 & 5.10 & 45.97 & 14.71 \\
70.27 & 6.80 & 67.13 & 23.46 \\
88.40 & 9.12 & 74.77 & 27.65 \\
96.90 & 12.44 & 114.77 & 46.44 \\
137.60 & 15.20 & 134.56 & 60.00 \\
167.55 & 19.30 & 169.23 & 82.07 \\
\hline
\end{tabular}

Using an optimization program in MATLAB, values for $\eta_{\infty}=26.5 \mathrm{mPa} \cdot \mathrm{s}$ and $R^{2}=-4.0 e-06$ are obtained which obviously show the worst fit to measurements. Figure 6 shows the apparent viscosity for which the Newtonian model has obviously failed to capture trends of the apparent viscosity due to non-Newtonian behavior of the WBM. The consistency curve of the Newtonian model has overpredicted measurements as seen in Figure 6.

6.3.2. Bingham Rheology Model. For the Bingham model, it is assumed that $K=0, n=1, \eta=\left(\tau_{0} / \dot{\gamma}\right)+\eta_{\infty}, \operatorname{Re}_{\text {gen Bingham }}=\rho$ $D \bar{u} /\left(\tau_{0} / 8(D / \bar{u})+\eta_{\infty}(3 m+1) / 4 m\right)$, and $m=\eta_{\infty}(8 \bar{u} / D) /\left(\tau_{0}\right.$ $\left.+\eta_{\infty}(8 \bar{u} / D)\right)$. Therefore, equation (28) needs two parameters, i.e., $\tau_{0}$ and $\eta_{\infty}$. The generalized optimization MATLAB program was then applied to investigate the suitability of the Bingham model for WBM examined here.

The Bingham rheology model has perfectly fitted WBM measurements (see Figure 7) with values $\tau_{0}=10.5 \mathrm{~Pa}, \eta_{\infty}$ $=8.8 \mathrm{mPa} \cdot \mathrm{s}$, and $R^{2}=1.0$.

As observed in Figure 7, the exact fit is obtained for TCV-measured apparent viscosity and shear stress with the values from the Bingham model.

6.3.3. Power Law (PL) Rheology Model. In the power law (PL) rheology model (Table 1), it is assumed that $\tau_{0}=0$, $\eta_{\infty}=0, \quad \eta=K \dot{\gamma}^{n-1}, \quad m=n, \quad$ and $\quad \operatorname{Re}_{\text {gen PL }}=\rho D^{n} \overline{\mathcal{u}}^{2-n} / K$ $[(3 m+1) / 4 m]^{n} 8^{n-1}$. This model needs two parameters, i.e., $K$ and $n$, to be found by the optimization program.

Power law (PL) has nicely fitted WBM measurements with $K=2.4826, n=0.2961$, and $R^{2}=0.9930$ (see Figure 8).

6.3.4. Herschel-Bulkley (HB) Rheology Model. The HerschelBulkley (HB) rheology model is a widely accepted model for drilling fluids. As shown in Table 1, the Herschel-Bulkley (HB) model is expressed by $\eta_{\infty}=0$ and $\eta=\left(\tau_{0} / \dot{\gamma}\right)+K \dot{\gamma}^{n-1}$ with 3 unknown parameters $\tau_{0}, K$, and $n$. The generalized Reynolds number is given by $\operatorname{Re}_{\text {gen HB }}=\rho D^{n} \bar{u}^{2-n} /\left(\tau_{0} / 8\right.$ $\left.(D / \bar{u})^{n}+K[(3 m+1) / 4 m]^{n} 8^{n-1}\right)$ in which $m=n K(8 \bar{u} / D)^{n} /($ $\left.\tau_{0}+K(8 \bar{u} / D)^{n}\right)$. The MATLAB optimization program was applied to find the best fit to experimental data using the HB model.

The Herschel-Bulkley (HB) rheology model has fitted very well WBM measurements (see Figure 9) with $\tau_{0}=$ 


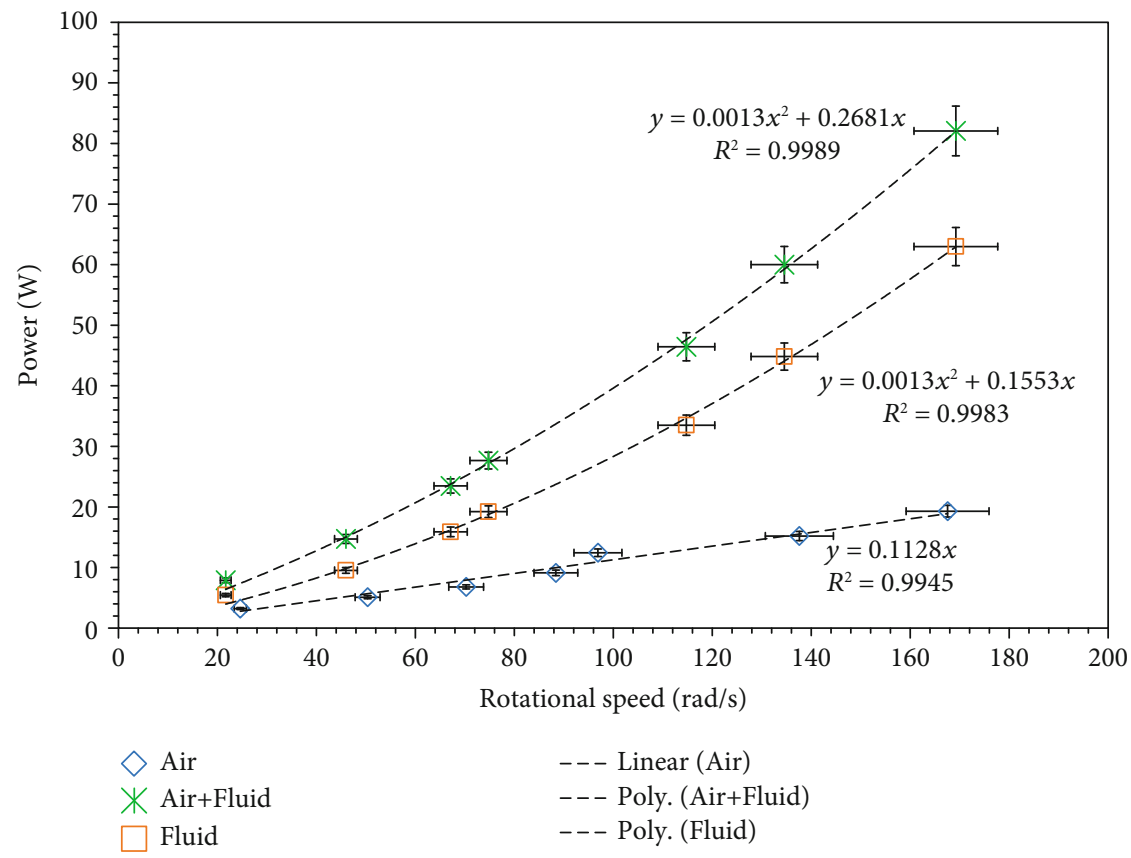

FIGURE 5: Power models in TCV: mechanical power (air), total power (fluid+air), and fluid power using WBM (4\% vol bentonite API standard).

9.6333 $\mathrm{Pa}, K=0.0325 \mathrm{~Pa} \cdot \mathrm{s}^{n}$, and $n=0.8234$ with an accuracy of $R^{2}=0.9995$.

6.3.5. Herschel-Bulkley-Extended (HBE) Rheology Model. The Herschel-Bulkley-Extended (HBE) rheology model is the most complete model compared with previous models. The HBE was introduced by Madlener et al. [32, 34] to study non-Newtonian gel fluids. The HBE model is expressed by $\eta=\left(\tau_{0} / \dot{\gamma}\right)+K \dot{\gamma}^{n-1}+\eta_{\infty}$ which needs four parameters $\tau_{0}, K$ , $n$, and $\eta_{\infty}$. HBE is seen as the generalized model for all models (see Table 1). The Reynolds number is $\operatorname{Re}_{\text {gen } \mathrm{HBE}}=$ $\rho D^{n} \bar{u}^{2-n} /\left(\tau_{0} / 8(D / \bar{u})^{n}+K[(3 m+1) / 4 m]^{n} 8^{n-1}+\eta_{\infty}(3 m+1) /\right.$ $\left.4 m(D / \bar{u})^{n-1}\right)$ in which $m=\left(n K(8 \bar{u} / D)^{n}+\eta_{\infty}(8 \bar{u} / D)\right) /\left(\tau_{0}+\right.$ $\left.K(8 \bar{u} / D)^{n}+\eta_{\infty}(8 \bar{u} / D)\right)$. In our in-house-developed MATLAB program, the HBE model can be reduced to any of the aforementioned 5 models to apply optimization and to obtain unknown parameters by fitting to experimental data. Here, we examine the HBE model for validation of experimental results for WBM measurements.

Bingham and HBE models produced a perfect match with WBM measurements (see Figure 10). The 4 parameters of the HBE model are obtained as $\tau_{0}=10.5 \mathrm{~Pa}, K=3.5720$ $\mathrm{Pa} \cdot \mathrm{s}^{n}, n=-1.9281$, and $\eta_{\infty}=8.8 \mathrm{mPa} \cdot \mathrm{s}$ with $100 \%$ accuracy of $R^{2}=1.0000$.

6.3.6. Four-Parameter Drilling Models. The Cross model is one of the 4-parameter models widely used for drilling fluids. This model was presented in [17] to study drilling fluids. The Cross model is expressed by $\eta=\eta_{\infty}+\left(\left(\eta_{0}-\eta_{\infty}\right.\right.$ )$\left./\left(1+(K \dot{\gamma})^{n}\right)\right)$ with 4 unknown parameters $\eta_{0}, K, n$, and $\eta_{\infty}$. In the developed MATLAB program, the Cross model is fitted for WBM (4\% vol bentonite API standard) measure- ments as illustrated in Figure 11. The 4 parameters of the Cross model are obtained as $\eta_{\mathrm{o}}=1316 \mathrm{mPa} \cdot \mathrm{s}, K=0.1084 \mathrm{~s}$ ,$n=1.0337$, and $\eta_{\infty}=9.0 \mathrm{mPa} \cdot \mathrm{s}$ with $100 \%$ accuracy of $R^{2}$ $=1.0000$.

The Carreau model is also one of the 4-parameter models widely used for drilling fluids [17]. The Carreau model is expressed by $\eta=\eta_{\infty}+\left(\left(\eta_{o}-\eta_{\infty}\right) /\left(1+(K \dot{\gamma})^{2}\right)^{-n / 2}\right)$ with 4 unknown parameters $\eta_{\mathrm{o}}, K, n$, and $\eta_{\infty}$. The Carreau model is fitted to WBM ( $4 \%$ vol bentonite API standard) measurements as illustrated in Figure 12. The 4 parameters of the Carreau model are obtained as $\eta_{\mathrm{o}}=1894.6 \mathrm{mPa} \cdot \mathrm{s}, K$ $=0.1360 \mathrm{~s}, \quad n=-1.0925$, and $\eta_{\infty}=10.6 \mathrm{mPa} \cdot \mathrm{s}$ with an accuracy of $R^{2}=0.9993$. This model is less accurate than the Cross model.

6.3.7. Friction Factor. Darcy friction factor calculations are presented here. For the Newtonian rheology model, the measurement values in the TCV fall in the turbulent region and deviate significantly from the laminar friction factor ( $f_{\text {laminar }}=64 / \mathrm{Re}$ ) (see Figure 13). The shift from the laminar friction factor exhibits turbulent flow condition for all speeds.

For the Bingham model, we found that the Darcy friction factor $\left(f_{\text {Darcy }}=8 \tau_{\text {wi }} / \rho \bar{u}_{\theta}^{2}\right)$ presented in [34] is more suitable for comparing TCV flows with the laminar friction factor $\left(f_{\text {laminar }}=64 / \mathrm{Re}\right)$. As shown in Figure 13, transition from laminar flow (solid line) occurred at ssss $_{\text {Bingham }}=$ 2000 and above, which is the laminar limit in pipe flows. The deviation from the laminar Darcy friction shows arrival in turbulent flow condition. Madlener and Ciezki [43, 44] also concluded that this deviation is an indication of turbulence. 
TABLE 4: Flow property measurement in TCV using WBM.

\begin{tabular}{lccccccccc}
\hline $\begin{array}{l}\text { Speed } \\
(\mathrm{RPM})\end{array}$ & $\begin{array}{c}\text { Total } \\
\text { power }(\mathrm{W})\end{array}$ & $\begin{array}{c}\text { Fluid } \\
\text { power }(\mathrm{W})\end{array}$ & $\begin{array}{c}\text { Fluid torque } \\
(\mathrm{N} \cdot \mathrm{m})\end{array}$ & $\begin{array}{c}\text { Shear rate } \\
(1 / \mathrm{s})\end{array}$ & $\begin{array}{c}\text { Shear } \\
\text { stress }(\mathrm{Pa})\end{array}$ & $\begin{array}{c}\text { Apparent } \\
\text { viscosity }(\mathrm{cP})\end{array}$ & $\begin{array}{c}\text { Average } \\
\text { velocity }(\mathrm{m} / \mathrm{s})\end{array}$ & $\begin{array}{c}\text { Reynolds } \\
\text { number }\end{array}$ & $\begin{array}{c}\text { Darcy } \\
\text { friction factor }\end{array}$ \\
\hline 206.99 & 7.89 & 3.98 & 0.1835 & 217.40 & 7.81 & 35.92 & 0.36 & $1.08 E+04$ & 0.4642 \\
439.00 & 14.71 & 9.89 & 0.2151 & 460.98 & 9.15 & 19.85 & 0.77 & $4.85 E+04$ & 0.1210 \\
641.00 & 23.46 & 16.28 & 0.2426 & 673.16 & 10.32 & 15.33 & 1.12 & $1.03 E+05$ & 0.0640 \\
714.00 & 27.65 & 18.88 & 0.2525 & 749.78 & 10.75 & 14.33 & 1.25 & $1.28 E+05$ & 0.0537 \\
1096.00 & 46.44 & 34.95 & 0.3045 & 1150.89 & 12.96 & 11.26 & 1.91 & $3.02 E+05$ & 0.0275 \\
1284.99 & 60.00 & 44.44 & 0.3302 & 1349.34 & 14.05 & 10.41 & 2.24 & $4.15 E+05$ & 0.0217 \\
1616.00 & 82.07 & 63.51 & 0.3753 & 1697.00 & 15.97 & 9.41 & 2.82 & $6.57 E+05$ & 0.0156 \\
\hline
\end{tabular}

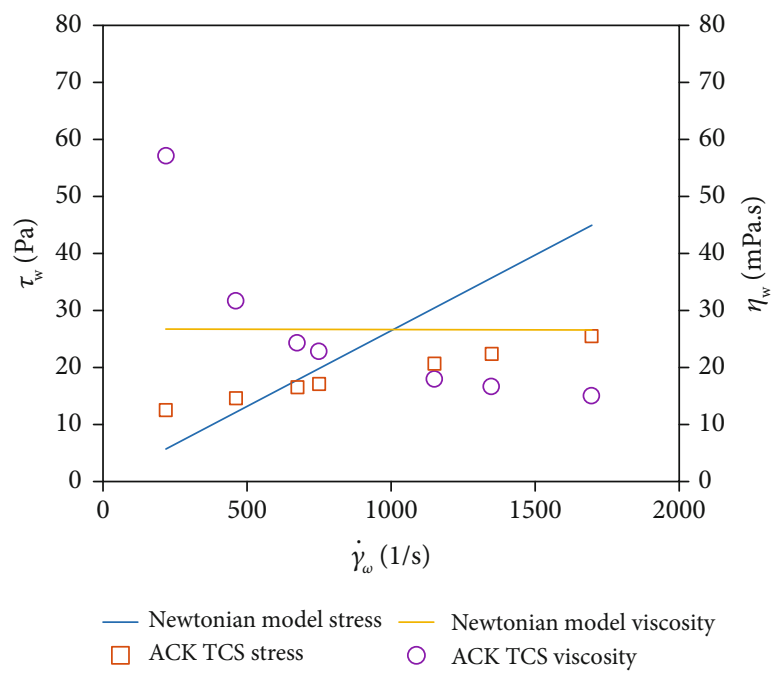

Figure 6: Newtonian model fit to WBM for the stress and the apparent viscosity.

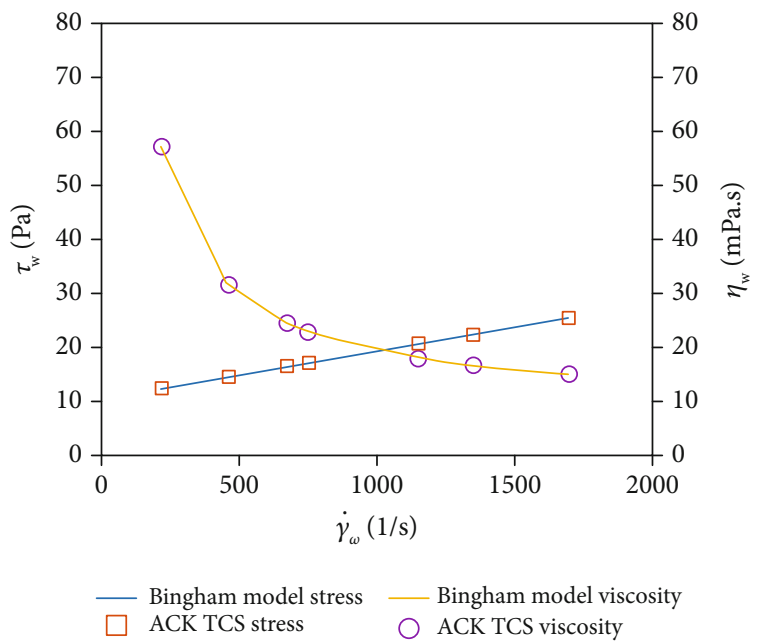

Figure 7: Bingham model fit to WBM with $\tau_{0}=10.5 \mathrm{~Pa}$ and $\eta_{\infty}$ $=8.8 \mathrm{mPa} \cdot \mathrm{s}$.

Using the power law (PL) for the WBM, it seems that the Darcy friction factor remains with a slight negative shift below the laminar friction factor for the full range of exper-

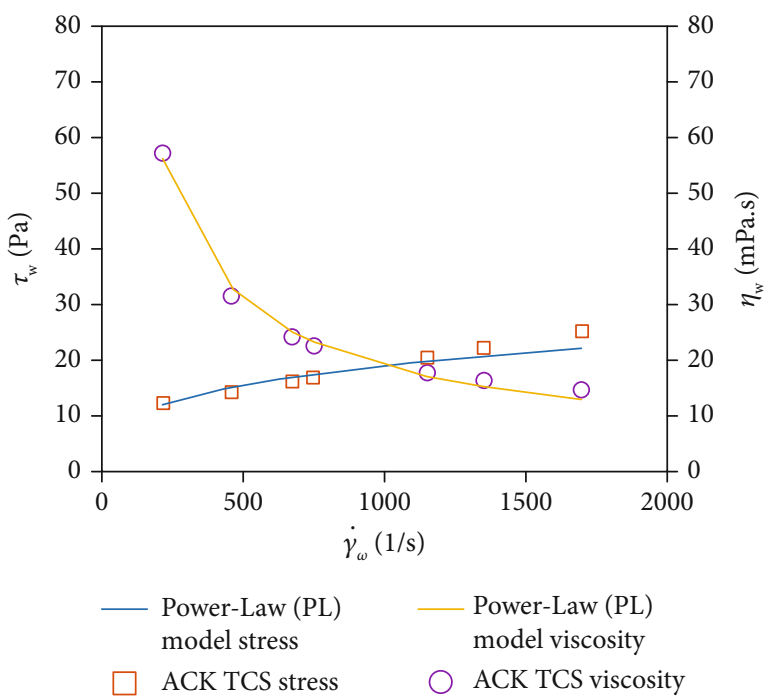

Figure 8: Power law (PL) model fit to WBM with $K=2.4826 \mathrm{~Pa} \cdot \mathrm{s}^{n}$ and $n=0.2961$.

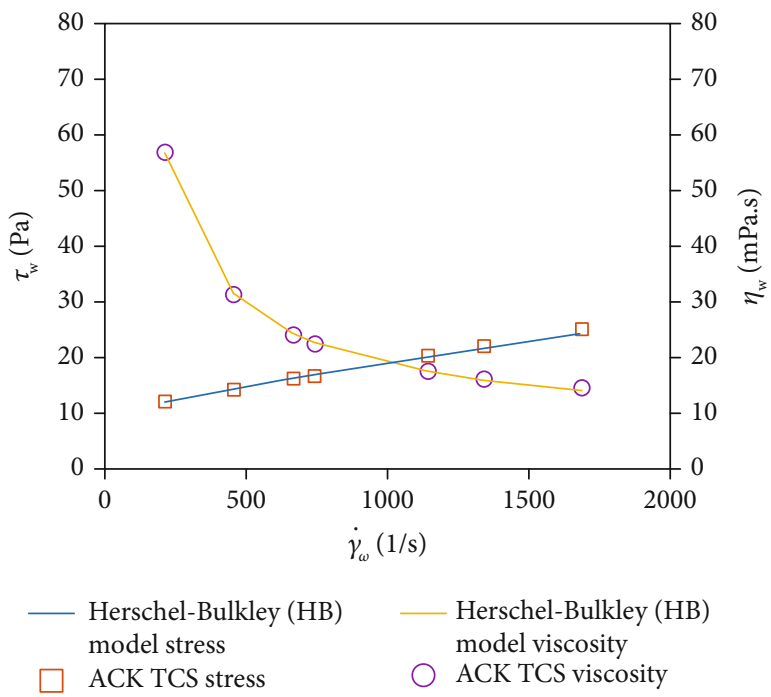

Figure 9: Herschel-Bulkley (HB) model for fitting WBM measurements.

imental data as shown in Figure 13. Madlener et al. [34] reported the negative deviation with no explanation for the reason of that. 


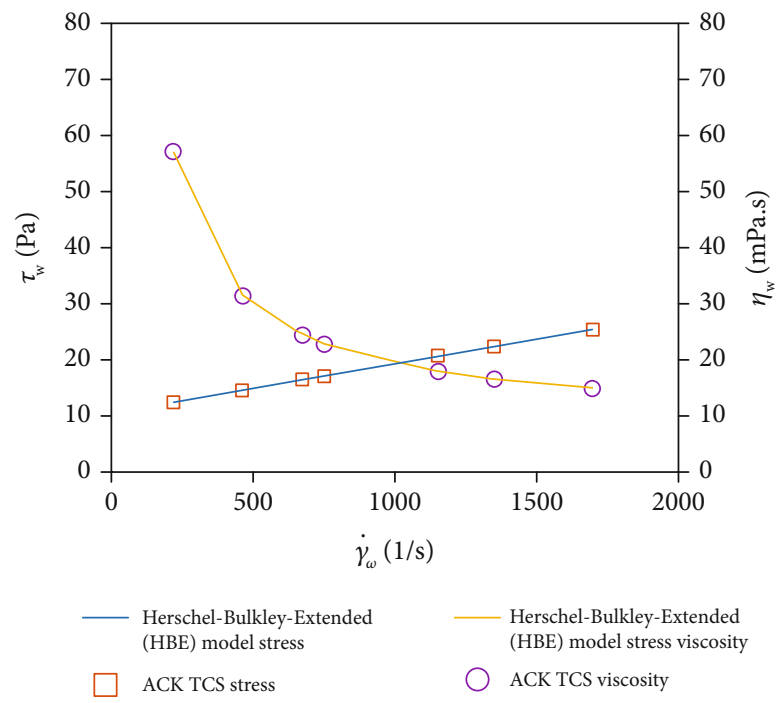

Figure 10: Herschel-Bulkley-Extended (HBE) rheology model fitting with WBM measurements.

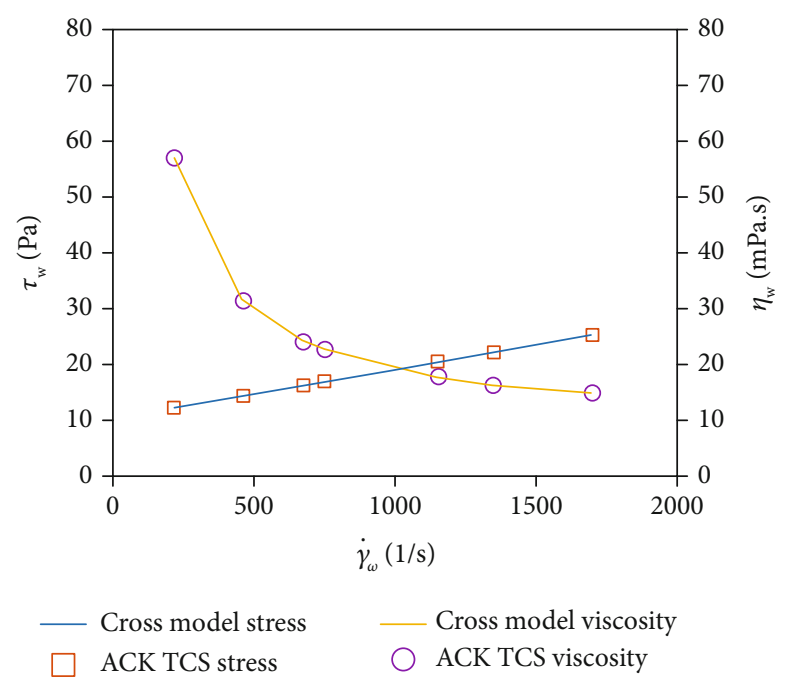

FIgURE 11: Cross model fit to WBM.

The HB model in comparison with the Bingham model (highest accuracy) shows that the measured values of the Darcy friction factor have deviated very slightly (negative deviation) from the laminar friction factor (see Figure 13).

For the HBE model, it is observed from the Darcy friction factor that laminar flow is deviated at the higher Reynolds number of around $\mathrm{Re}=2000$ compared with the Bingham model; this is the exact match with a classical upper limit of laminar flows in internal pipe flows. However, the Bingham model may be preferred over HBE due to its simplicity.

\section{Concluding Remarks and Future Works}

It is of interest to enhance mud properties particularly at high-speed drilling. In this work, a high-speed viscometer is developed using the classical Taylor-Couette system (TCV) with low volume capacity of $100 \mathrm{ml}$ to operate at speeds from

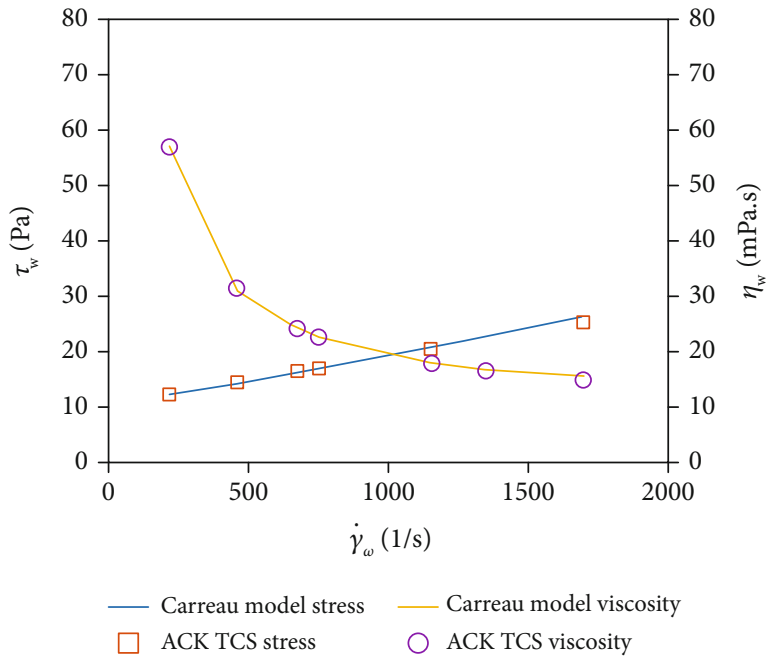

FIgURE 12: Carreau model fit to WBM.

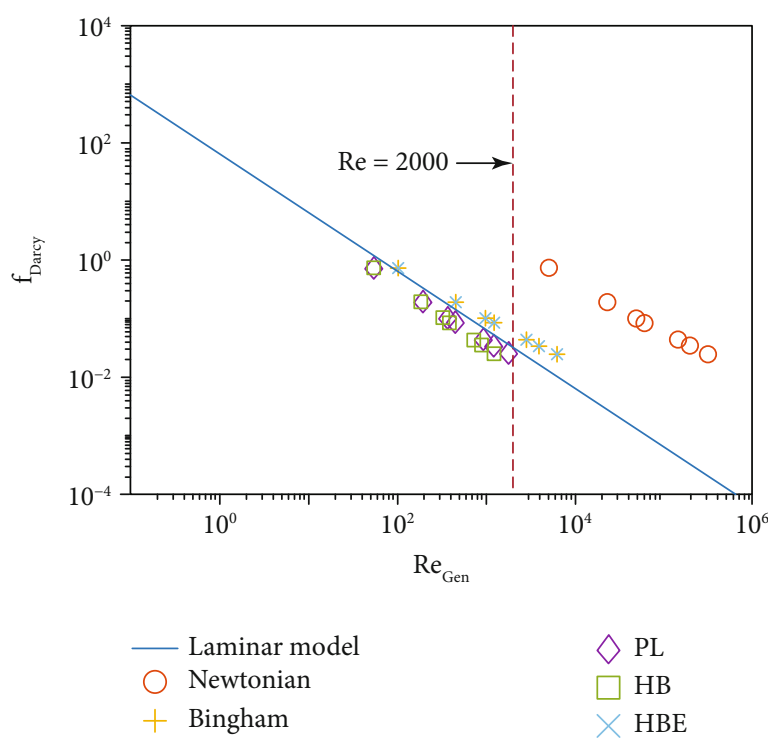

FIGURE 13: Darcy friction factor for all models versus the laminar model.

0 to 1600 RPM. Some standard viscometers merely operate at low speeds below 600 RPM. However, it is inevitable to study rheology of drilling fluids at turbulent flow conditions in a real drilling process. A high-speed TCV power-based system was developed to study rheology of WBM ( $4 \%$ vol bentonite API standard) at laminar to turbulent flow conditions. An optimization program was developed in MATLAB to fit WBM measurements with five classical rheology models extracted from the HBE model and two 4-parameter family models, i.e., Cross and Carreau models. The following conclusions are obtained:

(i) The modelled apparent viscosity and the shear stress are presented and discussed for all models. Bingham, HBE, and Cross models have fitted precisely with the experimental data 
(ii) A positive shift from the laminar friction factor was detected at higher Reynolds numbers than 2000 which indicates initiation of turbulent flows. It is also shown that the upper limit of laminar internal pipe flows $\left(\operatorname{Re}_{\mathrm{Gen}}=2000\right)$ may be valid for the annulus flow of the TCV

(iii) The optimization methodology developed here can feasibly obtain 4-parameter or more rheology models. This will be useful particularly for rheology model development of nanofluids

(iv) The HB and PL models exhibited the unresolved negative shift from the laminar friction factor to be further investigated

Work currently continues on WBM enhanced by nanoparticles. Nanoparticles are usually very expensive, so the designed ACK TCV with the volume of only $100 \mathrm{ml}$ is a good viscometer for studying nanofluids.

\section{Nomenclature}

$\begin{array}{ll}\text { API: } & \text { American Petroleum Institute } \\ \text { API RP 131: } & \text { API Recommended Practice for Laboratory } \\ & \text { Testing of Drilling Fluids } \\ \text { ACK: } & \text { Australian College of Kuwait } \\ \text { DC: } & \text { Direct current } \\ \text { FANN: } & \text { Company brand of viscometer } \\ \text { GS: } & \text { Gel strength } \\ \text { HB: } & \text { Herschel-Bulkley } \\ \text { HBE: } & \text { Herschel-Bulkley-Extended } \\ \text { HPHT: } & \text { High-Pressure-High-Temperature } \\ \text { LCD: } & \text { Liquid crystal display } \\ \text { MATLAB: } & \text { Matrix Laboratory (Software Company Inc.) } \\ \text { MCR: } & \text { Modular Compact Rheometer } \\ \text { OFITE: } & \text { A viscometer brand } \\ \text { OBM: } & \text { Oil-based mud } \\ \text { PL: } & \text { Power law } \\ \text { PV: } & \text { Plastic viscosity } \\ \text { pH: } & \text { Potential of hydrogen } \\ \text { RMSE: } & \text { Root mean square error } \\ \text { RPM: } & \text { Rotation per minute } \\ \text { TCS: } & \text { Taylor-Couette system } \\ \text { WBM: } & \text { Water-based mud } \\ \text { YP: } & \text { Yield point. } \\ & \end{array}$

\section{Data Availability}

Data are included in the manuscript. If other data is required, upon request, we will provide them. The figures and tables are modified to our best possible resolutions and updated here; we cannot do anything beyond this.

\section{Conflicts of Interest}

The authors declare that they have no known competing financial interests or personal relationships that could have appeared to influence the work reported in this paper.

\section{Acknowledgments}

We would like to greatly acknowledge KFAS (the Kuwait Foundation for the Advancement of Sciences) for providing the generous grant no. PR18-15EP-01 to conduct this research work. We wish to thank all ACK staff who assisted us in this research. We also are thankful to Dr. Daniel B. Echeverry for his support and advice on the TaylorCouette system.

\section{References}

[1] K. Almutairi, S. S. Hosseini Dehshiri, S. J. Hosseini Dehshiri, A. Mostafaeipour, A. Issakhov, and K. Techato, "A thorough investigation for development of hydrogen projects from wind energy: a case study," International Journal of Hydrogen Energy, vol. 46, no. 36, pp. 18795-18815, 2021.

[2] R. Kalbasi, M. Jahangiri, A. Mosavi et al., "Finding the best station in Belgium to use residential-scale solar heating, one-year dynamic simulation with considering all system losses: economic analysis of using ETSW," Sustainable Energy Technologies and Assessments, vol. 45, article 101097, 2021.

[3] A. Mostafaeipour, S. J. H. Dehshiri, S. S. H. Dehshiri, and M. Jahangiri, "Prioritization of potential locations for harnessing wind energy to produce hydrogen in Afghanistan," International Journal of Hydrogen Energy, vol. 45, no. 58, pp. 33169-33184, 2020.

[4] K. Almutairi, A. Mostafaeipour, E. Jahanshahi et al., "Ranking locations for hydrogen production using hybrid wind-solar: a case study," Sustainability, vol. 13, no. 8, article 4524, 2021.

[5] A. Mostafaeipour, S. J. Hosseini Dehshiri, and S. S. Hosseini Dehshiri, "Ranking locations for producing hydrogen using geothermal energy in Afghanistan," International Journal of Hydrogen Energy, vol. 45, no. 32, pp. 15924-15940, 2020.

[6] A. Mostafaeipour, S. J. Hosseini Dehshiri, S. S. Hosseini Dehshiri, M. Jahangiri, and K. Techato, "A thorough analysis of potential geothermal project locations in Afghanistan," Sustainability, vol. 12, no. 20, article 8397, 2020.

[7] A. Mostafaeipour, M. Jahangiri, A. Haghani et al., "Statistical evaluation of using the new generation of wind turbines in South Africa," Energy Reports, vol. 6, pp. 2816-2827, 2020.

[8] A. E. Bayat, P. Jalalat Moghanloo, A. Piroozian, and R. Rafati, "Experimental investigation of rheological and filtration properties of water-based drilling fluids in presence of various nanoparticles," Colloids and Surfaces A: Physicochemical and Engineering Aspects, vol. 555, pp. 256-263, 2018.

[9] E. R. Booser, CRC Handbook of Lubrication. Theory and Practice of Tribology: Volume II: Theory and Design, CRC Press LLC, 1984.

[10] R. P. Chhabra and J. F. Richardson, Non-Newtonian Flow in the Process Industries: Fundamentals and Engineering Applications, Butterworth-Heinemann, 1999.

[11] Z. Vryzas and V. C. Kelessidis, "Nano-based drilling fluids: a review," Energies, vol. 10, no. 4, p. 540, 2017.

[12] H. Mahmoud, A. Hamza, M. S. Nasser, I. A. Hussein, R. Ahmed, and H. Karami, "Hole cleaning and drilling fluid sweeps in horizontal and deviated wells: comprehensive review," Journal of Petroleum Science and Engineering, vol. 186, article 106748, 2020. 
[13] M. Ali, H. H. Jarni, A. Aftab et al., "Nanomaterial-based drilling fluids for exploitation of unconventional reservoirs: a review," Energies, vol. 13, no. 13, article 3417, 2020.

[14] K. Karakosta, A. C. Mitropoulos, and G. Z. Kyzas, "A review in nanopolymers for drilling fluids applications," Journal of Molecular Structure, vol. 1227, article 129702, 2021.

[15] R. E. Swai, "A review of molecular dynamics simulations in the designing of effective shale inhibitors: application for drilling with water-based drilling fluids," Journal of Petroleum Exploration and Production Technology, vol. 10, no. 8, pp. 35153532, 2020.

[16] Y. H. Chai, S. Yusup, and V. S. Chok, "A review on nanoparticle addition in base fluid for improvement of biodegradable ester-based drilling fluid properties," Chemical Engineering Transactions, vol. 45, pp. 1447-1452, 2015.

[17] S. N. Shah, N. H. Shanker, and C. C. Ogugbue, "Future challenges of drilling fluids and their rheological measurements," in AADE Fluids Conference and Exhibition, Houston, TX, USA, 2010.

[18] M. Amanullah and A. M. Al-Tahini, "Nano-technology-its significance in smart fluid development for oil and gas field application," in SPE Saudi Arabia Section Technical Symposium, AlKhobar, Saudi Arabia, May 2009.

[19] Z. Tang and S. Li, "A review of recent developments of friction modifiers for liquid lubricants (2007-present)," Current Opinion in Solid State and Materials Science, vol. 18, no. 3, pp. 119139, 2014.

[20] M. F. Fakoya and R. M. Ahmed, "A generalized model for apparent viscosity of oil-based muds," Journal of Petroleum Science and Engineering, vol. 165, pp. 777-785, 2018.

[21] R. Kumar, V. Kumar, D. K. Rajak, and C. Guria, “An improved estimation of shear rate using rotating coaxial-cylinder Fann viscometer: a rheological study of bentonite and fly ash suspensions," International Journal of Mineral Processing, vol. 126, pp. 18-29, 2014.

[22] E. Podryabinkin, V. Rudyak, A. Gavrilov, and R. May, "Detailed modeling of drilling fluid flow in a wellbore annulus while drilling," in Proceedings of the ASME 2013 32nd International Conference on Ocean, Offshore and Arctic Engineering. Volume 6: Polar and Arctic Sciences and Technology; Offshore Geotechnics; Petroleum Technology Symposium, Nantes, France, June 2013.

[23] M. Couette, "Studies relating to the friction of liquids," Annales de chimie et de physique, vol. 21, pp. 433-510, 1890.

[24] G. I. Taylor, "VIII. Stability of a viscous liquid contained between two rotating cylinders," Philosophical Transactions of the Royal Society of London Series A, Containing Papers of a Mathematical or Physical Character, vol. 223, pp. 289-343, 1923.

[25] D. P. M. van Gils, Highly Turbulent Taylor-Couette Flow, University of Twente, Enschede, The Netherlands, 2011.

[26] C. D. Andereck, S. S. Liu, and H. L. Swinney, "Flow regimes in a circular Couette system with independently rotating cylinders," Journal of Fluid Mechanics, vol. 164, pp. 155-183, 1986.

[27] S. Chandrasekhar, Hydrodynamic and Hydromagnetic Stability, Courier Corporation, 2013.

[28] P. G. Drazin and W. H. Reid, Hydrodynamic Stability, Cambridge University Press, 2004.

[29] D. B. Echeverry, Taylor-Couette Flow Cell, AAPT Advanced Labs, Willamette University, 2019.
[30] P. Chossat and G. Iooss, The Couette-Taylor Problem, Springer Science \& Business Media, 2012.

[31] M. M. Vitturi, "Navier-Stokes Equations in Cylindrical Coordinates," 2020, https://demichie.github.io/NS_cylindrical/.

[32] H.-S. Dou, B. C. Khoo, and K. S. Yeo, "Instability of TaylorCouette flow between concentric rotating cylinders," International Journal of Thermal Sciences, vol. 47, pp. 1422-1435, 2008.

[33] A. Kristensen, "Flow properties of water-based drilling fluids," in Institutt for petroleumsteknologi og anvendt geofysikk, NTNU - Trondheim, Norwegian University of Science and Technology, 2013.

[34] K. Madlener, B. Frey, and H. Ciezki, "Generalized Reynolds number for non-Newtonian fluids," Progress in Propulsion Physics, vol. 1, pp. 237-250, 2009.

[35] R. Caenn, H. C. Darley, and G. R. Gray, Composition and Properties of Drilling and Completion Fluids, Gulf Professional Publishing, 2011.

[36] J. L. Devore, Probability and Statistics for Engineering and the Sciences, Cengage Learning, 2015.

[37] K. Pearson, "Notes on regression and inheritance in the case of two parents," Proceedings of the Royal Society of London, vol. 58, pp. 240-242, 1895.

[38] A. S. Masoud Rashidi, B. Misbah, M. Sabati, and K. Vaidyan, Developing a Taylor-Couette Stand-Alone Viscometer for Testing Drilling Fluids with Turbulent Nano Fluid Flow and Testing Stability of Different Shale Materials, Kuwait Foundation for the Advancement of Science (KFAS), 2020, Grant No. PR1815EP-01.

[39] M. Rashidi, A. Sedaghat, B. Misbah, M. Sabati, and K. Vaidyan, "Experimental study on energy saving and friction reduction of $\mathrm{Al}_{2} \mathrm{O}_{3}$-WBM nanofluids in a high-speed Taylor-Couette flow system," Tribology International, vol. 154, article 106728, 2021.

[40] J. Fink, Petroleum Engineer's Guide to Oil Field Chemicals and Fluids, Gulf Professional Publishing, 2015.

[41] A. G. Iscan and M. V. Kok, "Effects of polymers and CMC concentration on rheological and fluid loss parameters of waterbased drilling fluids," Energy Sources, Part A, vol. 29, no. 10, pp. 939-949, 2007.

[42] 13B-1 AR, Recommended Practice for Field Testing WaterBased Drilling Fluids, API, 4th edition, 2009.

[43] K. Madlener and H. Ciezki, "Analytical description of the flow behavior of extended Herschel-Bulkley fluids with regard to gel propellants," in Energetic Materials - Performance und Safety, 186-1-186-13. 36th International Annual Conferece of ICT, p. 186-1, Karlsruhe, Germany, 2005.

[44] K. Madlener and H. Ciezki, "Theoretical Investigation of the Flow Behavior of Gelled Fuels of the Extended Herschel Bulkley Type," in 1st European Conference for Aero-Space Sciences (EUCASS2005), Moscow, Russia, 2005. 